\title{
ВЛИЯНИЕ РАЗЛИЧНЫХ МЕТОДОВ ЭНДОХИРУРГИЧЕСКОГО ГЕМОСТАЗА НА ОВАРИАЛЬНЫЙ РЕЗЕРВ ПРИ ЭНУКЛЕАЦИИ ЭНДОМЕТРИОИДНЫХ КИСТ У ПАЦИЕНТОК ПОЗДНЕГО РЕПРОДУКТИВНОГО ВОЗРАСТА
}

\author{
Соколова Е.И. ${ }^{1}$, Куценко И.И. ${ }^{1}$, Кравцова Е.И. ${ }^{1}$, Томина О.В. ${ }^{1}$ \\ ${ }^{1}$ Федеральное государственное бюджетное образовательное учреждение высшего образования «Кубанский \\ государственный медицинский университет» Министерства здравоохранения Российской Федерации, \\ Kраснодap, e-mail: corpus@ksma.ru
}

В результате до- и послеоперационного обследования 109 пациенток позднего репродуктивного возраста с впервые выявленными эндометриоидными кистами яичника изучена динамика параметров системы перекисного окисления липидов, антиоксидантной защиты и изменений в цитокиновом балансе перитонеальной жидкости, полученная в результате лапароскопической энуклеации эндометриоидных кист яичника с выполнением интраоперационного гемостаза биполярным электродом, аргоноплазменной коагуляцией и рассасывающимся гемостатическим материалом. Оценено влияние различных методов интраоперационного гемостаза на овариальный резерв яичников у пациенток в позднем репродуктивном возрасте. Показано, что при использовании энергозависимого интраоперационного гемостаза на фоне регуляторной дисфункции, определяемой инертностью выработки IL2, на местном уровне нарушается сбалансированность функционального состояния межклеточных иммуномодуляторов IL6, TNFa иx антагониста ИЛ-10, что ведет к усилению проапоптических влияний и замедлению регенеративно-восстановительных реакций. Выявлено, что усугубление дисбаланса в системе цитокинов и ПОЛ/АОЗ оказывает негативное влияние на овариальный резерв. Данный процесс наиболее выражен при применении коагуляции биполярным электродом. Наименьшие сдвиги наблюдаются при применении интраоперационного гемостатика в виде рассасывающегося материала, изготовленного по методу контролируемого окисления восстановленной целлюлозы.

Ключевые слова: эндометриоидные кисты яичников, интраоперационный гемостаз, поздний репродуктивный возраст, овариальный резерв, цитокиновый статус, перекисное окисление липидов, антиоксидантная защита

\section{THE EFFECT OF VARIOUS METHODS OF ENDOSURGICAL HEMOSTASIS ON THE OVARIAN RESERVE IN CASE OF ENUCLEATION OF ENDOMETRIOMAS IN PATIENTS OF LATE REPRODUCTIVE AGE.}

\author{
Sokolova E.I. ${ }^{1}$, Kutsenko I.I. ${ }^{1}$, Kravtsova E.I. ${ }^{1}$, Tomina O.V. ${ }^{1}$ \\ ${ }^{I}$ Federal State Budgetary Educational Institution Of Higher Education «Kuban State Medical University» Of The \\ MinistryOf Healthcare Of The Russian Federation, Krasnodar,e-mail:corpus@ksma.ru
}

Pre- and postoperative assessment of 109 patients of advanced reproductive age with newly diagnosed cystic ovarian endometriosis, stimulated progression study of the parameters of the system of lipid peroxidation, antioxidant defense and changes in the cytokine balance of the peritoneal fluid, obtained in the result of laparoscopic enucleation of cystic ovarian endometriosis with achieving intraoperative hemostasis by a bipolar electrode, argon plasma coagulation and resorbable hemostatic material. The effect of various methods of intraoperative hemostasis on the ovarian reserve in patients of advanced reproductive age was assessed. It is shown that when using energy-dependent intraoperative hemostasis in the setting of regulatory dysfunction determined by the inertia of IL2 production, the balance of the functional state of the intercellular immunomodulators IL6, TNFa of their antagonist IL-10 is disturbed at the local level, which increases the proapoptotic effects and slowers regenerative rehabilitation. It has been proved that exacerbation of imbalance in the system of cytokines and «POL-AOD» has a negative effect on the ovarian reserve. This process is most significant when bipolar electrode coagulates. Least significant changes are observed with the use of the intraoperative hemostatic agent in the form of resorbable material, made according to the method of controlled oxidation of reduced cellulose.

Keywords: ovarian endometriomas, intraoperative hemostasis, late reproductive age, an ovarian reserve, cytokine status, lipid peroxidation, antioxidant defense

\section{Среди гинекологической патологии эндометриоидные кисты яичников (ЭКЯ)}


ведущих мест [1-3]. При этом даже при небольших размерах ЭКЯ у пациенток может наблюдаться снижение овариального резерва [3], причиной снижения которого в том числе является теория «выгорания» (burnout theory). Известно, что процесс снижения овариального резерва связан с атрезией на стадии малых антральных фолликулов, преимущественно посредством апоптоза. Причиной апоптоза и некроза примордиальных фолликулов при ЭКЯ и как следствие снижения овариального резерва является исходный оксидативный стресс, влияющий в том числе и на здоровую ткань яичника [4]. Многочисленными исследованиями было доказано, что генитальный эндометриоз сопровождается исходным дисбалансом цитокинов как периферической крови, так и перитонеальной жидкости, с преобладанием провоспалительного компонента и усилением проапоптического влияния [5, 6] и связь процессов в системе перекисного окисления липидов и антиоксидантной защиты (ПОЛ/АОЗ) и характеристик цитокинового статуса. Предполагается, что цитокиновый дисбаланс является одним из механизмов реализации оксидантного стресса, протекающего в дальнейшем с дестабилизацией липопероксидации и антиокислительной активности [6]. В случае необходимости хирургической операции (энуклеация кисты) важнейшей задачей является сохранение овариального резерва, тем более в позднем репродуктивном возрасте, с целью сохранения желанной фертильности и женского здоровья. Однако при использовании малоинвазивных технологий основным средством достижения гемостаза стали различные виды электрохирургического воздействия (что не может не приводить к дополнительному негативному воздействию на овариальную ткань), которые сопровождаются некрозом тканей, избыточным белковым катаболизмом и выраженным апоптозом клеток здоровой ткани яичника с развитием эндотоксикоза в той или иной степени в зависимости от используемых энергий. Поэтому поиск технологий интраоперационного гемостаза, минимально влияющих на систему ПОЛ/АОЗ и цитокиновом балансе и как следствие сохраняющих овариальный резерв, в том числе и у женщин позднего репродуктивного возраста, является одной из актуальных задач современной гинекологии.

Цель. Изучить влияние на овариальный резерв токсических метаболитов и изменений в цитокиновом балансе перитонеальной жидкости, полученных в результате различных методов эндохирургического гемостаза при энуклеации эндометриоидной кисты яичников у пациенток в позднем репродуктивном возрасте.

Материал и методы исследования. Обследованы 109 пациенток, прооперированных по поводу односторонней эндометриоидной кисты яичника. Всем им произведена эндохирургическая цистэктомия в плановом порядке. Размеры кист колебались от 40 до 60 мм (48,6 6 7,1 мм). В качестве адъювантной терапии все пациентки применяли диеногест 2 мг в сутки в течение всего периода наблюдения и далее до 6-9 месяцев в зависимости от 
репродуктивных планов. В соответствии с целью исследования пациентки были разделены на 3 клинические группы в зависимости от метода интраоперационного гемостаза. Пациенткам 1-й группы проведена биполярная коагуляция сосудов ложа кисты, пациенткам 2-й группы - аргоноплазменная коагуляция, 3-й группы - ложе кисты коагулировано с помощью применения рассасывающегося гемостатического материала, изготовленного по методу контролируемого окисления восстановленной целлюлозы. Группой контроля явились 35 условно здоровых женщин, поступивших на хирургическую стерилизацию. Определение принадлежности к стадии репродуктивного возраста для контрольной группы проводилось по критериям Рабочей группы по стадиям старения репродуктивной системы (STRAW+10).

Включение пациенток в клиническое исследование проводилось методом случайной выборки после получения информированного согласия и протоколировалось по стандартам Этического комитета Российской Федерации.

Из исследования исключались женщины с миомой матки, с рецидивом эндометриоидной кисты, с доброкачественными опухолями яичников другой этиологии или подозрением на малигнизацию образования, а также с наличием тяжелой соматической патологии либо другой экстрагенитальной патологии в стадии обострения.

Перед операцией определяли фолликулярный резерв по уровню антимюллерова гормона с помощью соответствующих тест-систем на иммунохимическом анализаторе «Access/DxI». Проводили исследование органов малого таза с определением размера кисты при помощи трансвагинального конвексного датчика на аппарате экспертного класса на аппаратах «Toshiba Aplio 500» с частотой 5 мГц и 6,5 мГц в режимах серой шкалы.

Во время операции и в первые сутки послеоперационного периода проводили забор перитонеальной жидкости, в которой определяли уровни содержания цитокинов (TNF-a, IL6, IL2, IL10, TNF- $\alpha$ ) методом ИФА (ASCENT, Финляндия) и использованием панели моноклональных антител (ЗАО ВЕКТОР-БЕСТ, г. Ростов-на-Дону) расчетом провоспалительного индекса (ПВИ) как отношение IL6/IL10 (у.е). Содержание токсических метаболитов (диеновых конъюгатов (ДК) ненасыщенных жирных кислот, малонового диальдегида (МДА) и молекул средней массы (МСМ)) и антиоксидантная система (активности супероксиддисмутазы (СОД)) перитонеальной жидкости оценивались по соответствующим методикам. Рассчитывался коэффициент, определяющий баланс в системе ПОЛ/АОЗ, как отношение МДА/СОД.

Статистический анализ полученных данных проводился с использованием пакета анализа Microsoft Excel 2000 и программы «STATISTICA» 6,0. Характеристики пациентов сравнивались посредством непарного $\mathrm{t}$-критерия Стьюдента, корреляция Спирмена использовалась для оценки соотношения между переменными. 


\section{Результаты исследования и их обсуждение}

Группы исследования были практически однородны по клинико-анамнестическому

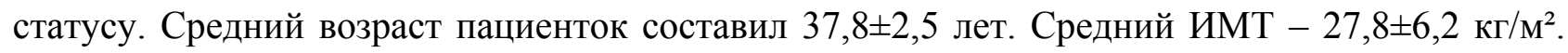
Планировали наступление беременности 32 пациентки (29,4\%). Первичное бесплодие выявлено у 12 (11\%), вторичное - у 16 (14,7\%) пациенток.

Полученные результаты сравнительного анализа динамики количественного содержания первичных, вторичных и конечных продуктов перекисного окисления липидов (ПОЛ) и системы антиоксидантной защиты (AO3) перитонеальной жидкости показали, что в раннем послеоперационном периоде на фоне исходного дисбаланса системы ПОЛ/АОЗ [7] наблюдается резкое возрастание уровней как первичных (диеновые конъюгаты), так и вторичных (малоновый диальдегид) молекулярных продуктов перекисного окисления липидов, а также молекул средней массы, в целом определяющих степень эндогенной

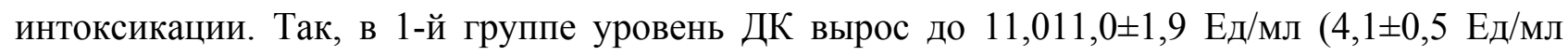

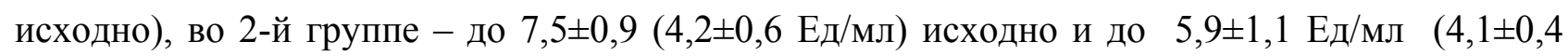

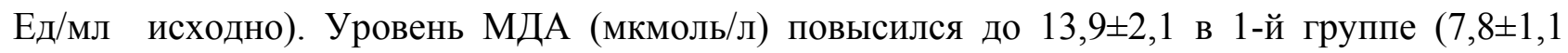
исходно, до 9,9 $\pm 1,0$ (исходно $7,7 \pm 1,2$ ) во 2-й группе и до $8,8 \pm 0,5$ (исходно $7,8 \pm 1,0$ ) у пациенток 3-й группы. Параллельно в первые сутки послеоперационного периода мы отметили в перитонеальной жидкости снижение активности СОД: в 1-й группе 2,3 $\pm 0,7$

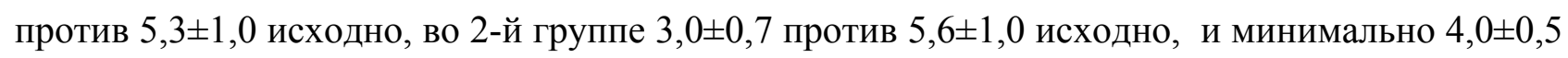
против 5,4 $\pm 0,9$ в 3-й группе. Соответственно во всех клинических группах определялось повышение соотношения МДА/СОД (у.е), характеризующее возрастание «окислительного потенциала» перитониальной жидкости. Данное соотношение возросло в 4,4 раза в 1-й группе, в 2,4 раза во 2-й группе и только в 1,3 раза в группе 3 (гемостатический материал) (табл. 1).

Таблица 1

Динамика показателей системы ПОЛ/АОС в перитонеальной жидкости в зависимости от метода эндохирургической коагуляции, $\mathrm{M} \pm \mathrm{m}$

\begin{tabular}{|c|c|c|c|c|c|c|}
\hline \multicolumn{2}{|l|}{ Показатели } & ДК, Ед/ мл & $\begin{array}{l}\text { МДА, } \\
\text { мкмоль/л }\end{array}$ & $\begin{array}{l}\text { СОД, } \\
\text { ед/мг }\end{array}$ & $\begin{array}{l}\text { МДА/СО } \\
\text { Д, у.е }\end{array}$ & $\begin{array}{l}\text { МСM, } \\
\text { E280,y.e } \\
\text { ОП }\end{array}$ \\
\hline \multirow[t]{2}{*}{$\begin{array}{l}\text { Биполярная } \\
\text { коагуляция }\end{array}$} & $\begin{array}{l}\text { До } \\
\text { операции }\end{array}$ & $4,1 \pm 0,5$ & $7,8 \pm 1,1$ & $5,3 \pm 1,0$ & $1,5 \pm 0,9$ & $\begin{array}{l}0,38 \pm 0,0 \\
9\end{array}$ \\
\hline & 1-е сутки & $11,0 \pm 1,9 * \wedge$ & $13,9 \pm 2,1 *$ & $2,3 \pm 0,7 * \wedge$ & $6,6 \pm 2,4 * \wedge$ & $\underset{\wedge}{0,8 \pm 0,1 *}$ \\
\hline \multirow[t]{2}{*}{$\begin{array}{l}\text { Аргоноплазмен } \\
\text { ная коагуляция }\end{array}$} & $\begin{array}{l}\text { До } \\
\text { операции }\end{array}$ & $4,2 \pm 0,6$ & $7,7 \pm 1,2$ & $5,6 \pm 1,0$ & $1,4 \pm 0,9$ & $0,41 \pm 0,9$ \\
\hline & 1-е сутки & $7,5 \pm 0,9 * \wedge$ & $9,9 \pm 1,0 * \wedge$ & $3,0 \pm 0,7 * \wedge$ & $3,4 \pm 0,8^{* \wedge}$ & $\begin{array}{l}0,6 \pm 0,1 * \\
\wedge\end{array}$ \\
\hline
\end{tabular}




\begin{tabular}{l|l|l|l|l|l|l|}
$\begin{array}{l}\text { Применение } \\
\text { гемостатическо } \\
\text { го материала }\end{array}$ & $\begin{array}{l}\text { Оо } \\
\text { операции }\end{array}$ & $4,1 \pm 0.4$ & $7,8 \pm 1,0$ & $5,4 \pm 0,9$ & $1,65 \pm 0,9$ & $0,38 \pm 0,9$ \\
\hline 1-е сутки & $5,9 \pm 1,1^{* \wedge}$ & $8,8 \pm 0,5^{* \wedge}$ & $4,0 \pm 0,5^{* \wedge}$ & $2,2 \pm 1,7^{* \wedge}$ & $0,4 \pm 0,1^{\wedge}$ \\
\hline Группа контроля, $\mathbf{n = 3 5}$ & $0,65 \pm 1,1$ & $3,6 \pm 0,8$ & $7,5 \pm 0,6$ & $0,5 \pm 0,02$ & $\begin{array}{l}0,03 \pm 0,0 \\
2\end{array}$ \\
\hline
\end{tabular}

где символ «*» соответствует статистически значимому различию показателей в послеоперационном периоде по сравнению со средними значениями аналогичных показателей до операции $(\mathrm{p}<0,005)$.

где символ «^» соответствует статистически значимому межгрупповому различию $(\mathrm{p}<0,005)$.

При анализе результатов динамики уровня цитокинов в перитонеальной жидкости выявлено возрастание провоспалительного потенциала, выражающееся в достоверном повышении концентрации IL 6 и TNF- $\alpha$ во всех клинических группах. При этом максимальный прирост как IL 6, так и TNF- $\alpha$ наблюдался в 1-й группе (при проведении биполярной коагуляции (65,8 $\pm 4,3$ против $28,6 \pm 5,3$ исходно) и $(9,5 \pm 0,9$ против $7,1 \pm 1,6$

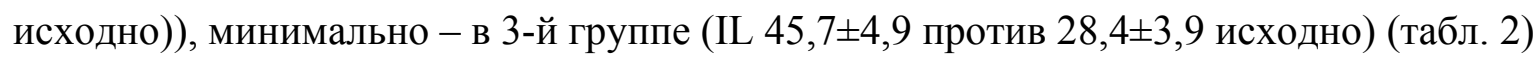

Таблица 2

Динамика показателей цитокинового статуса перитонеальной жидкости в зависимости от метода эндохирургической коагуляции, $\mathrm{M} \pm \mathrm{m}$

\begin{tabular}{|c|c|c|c|c|c|}
\hline \multicolumn{2}{|l|}{ Показатели } & \multirow{2}{*}{$\begin{array}{l}\text { IL } 10 \\
\text { Пкг/мл } \\
10,2 \pm 1,4\end{array}$} & \multirow{2}{*}{$\begin{array}{l}\text { IL } 6 \\
\text { пкг/мл } \\
28,6 \pm 5,3\end{array}$} & \multirow{2}{*}{$\begin{array}{l}\text { IL 2, } \\
\text { пкг/мл } \\
6,5 \pm 2,1\end{array}$} & \multirow{2}{*}{$\begin{array}{l}\text { TNF- } \alpha, \\
\text { пкг/мл } \\
7,1 \pm 1,6\end{array}$} \\
\hline Биполярная & До операции & & & & \\
\hline & 1-е сутки & $10,1 \pm 1,9$ & $65,8 \pm 4,3^{* \wedge}$ & $7,2 \pm 0,9$ & $9,5 \pm 0,9 * \wedge$ \\
\hline \multirow{2}{*}{$\begin{array}{l}\text { Аргоноплазменная } \\
\text { коагуляция, } n=35\end{array}$} & До операции & $9,9 \pm 0,9$ & $27,9 \pm 4,6$ & $6,5 \pm 1,6$ & $7,0 \pm 1,8$ \\
\hline & 1-е сутки & $11,16 \pm 1,4$ & $56,5 \pm 6,8 * \wedge$ & $7,52 \pm 0,9$ & $9,2 \pm 0,7 * \wedge$ \\
\hline \multirow{2}{*}{$\begin{array}{l}\text { Применение } \\
\text { гемостатического } \\
\text { материала, } \mathbf{n = 3 2}\end{array}$} & До операции & $10,3 \pm 0,8$ & $28,4 \pm 3,9$ & $6,6 \pm 1,6$ & $7,1 \pm 0,7$ \\
\hline & 1-е сутки & $12,3 \pm 2,04$ & $45,7 \pm 4,9 * \wedge$ & $7,3 \pm 0,9$ & $9,05 \pm 0,7 * \wedge$ \\
\hline \multicolumn{2}{|c|}{ Группа контроля, n=35 } & $9,2 \pm 1,4$ & $10,8 \pm 2,8$ & $4,4 \pm 1,6$ & $1,9 \pm 0,7$ \\
\hline
\end{tabular}

где символ «*» соответствует статистически значимому различию показателей в послеоперационном периоде по сравнению со средними значениями аналогичных показателей до операции $(\mathrm{p}<0,005)$.

где символ «^» соответствует статистически значимому межгрупповому различию $(\mathrm{p}<0,005)$.

Отставание противовоспалительного характеризовалось отсутствием динамики уровня IL10 и выражалось в достоверном возрастании провоспалительного индекса во всех клинических группах (p<0,005): в 1-й группе в 3 раза, во 2-й в 1,8 раза и в 3-й в 1,5 раза (рис. $1)$. 


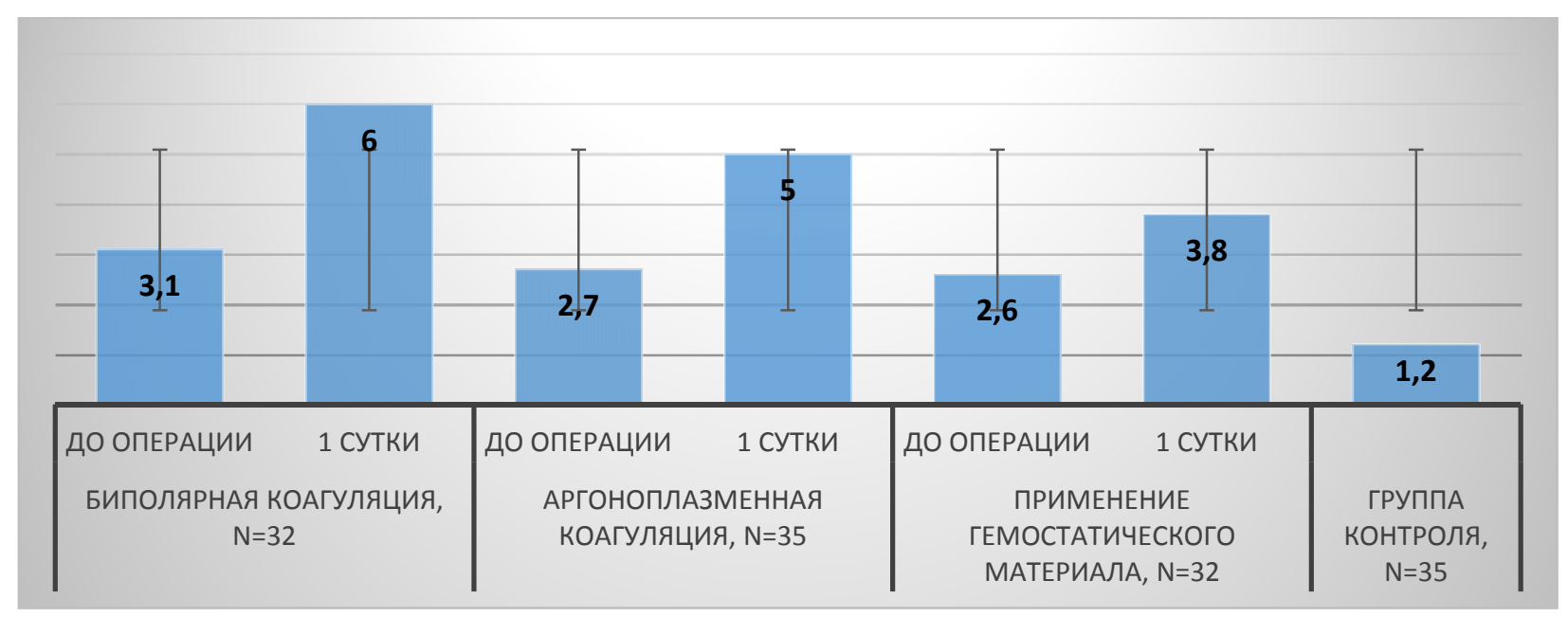

Рис. 1. Динамика ПВИ (IL6/IL10) перитонеальной жидкости в зависимости от метода эндохирургической коагулящии, $M \pm m$

Полученные результаты показали тесную взаимосвязь процессов перекисного окисления липидов и системы антиоксидантной защиты с цитокиновым профилем в перитонеальной жидкости больных с ЭКЯ, поскольку все реакции иммунитета в той или иной степени кислородозависимы. Это подтверждается статистически значимыми сильными положительными корреляционными взаимосвязями между процентами повышения IL6, TNF- $\alpha$ и процентом прироста коэффициента МДА/СОД во всех клинических группах. Коэффициент корреляции составил для IL6 и МДА: r=0,82 - в 1-й группе, r=0,89 - во 2-й группа и r=0,78 - в 3-й группе и для TNF- $\alpha(\mathrm{p}<0,005)$ : $\mathrm{r}=0,87$ - в 1-й группе $, \mathrm{r}=0,82$ - во 2-й группе и $\mathrm{r}=0,81$ - в 3-й группе.

Исследование овариального резерва, определяемого в нашем исследовании по уровню АМГ (негормонозависимый показатель), показало, что антимюллеров гормон Г в сыворотке крови значительно снизился после операции во всех группах независимо от того, какой метод гемостаза был применен, и через три месяца ни в одной из клинических групп не вернулся к дооперационным значениям. При средних дооперационных параметрах,

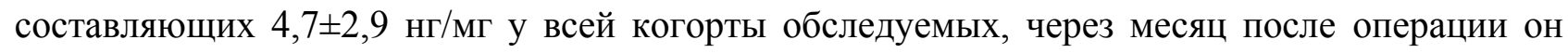

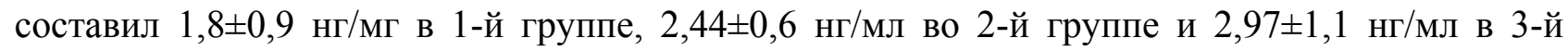
группе, $\mathrm{p}<0,005$ по всем группам. Максимально уровень АМГ снизился при применении во время цистэктомии коагуляции биполярным электродом. При применении гемостатического материала снижение уровня АМГ было минимальным. К третьему месяцу послеоперационного периода при условии выполнения аргоноплазменной коагуляции и применения гемостатического материала уровень АМГ прирастал до 2,8

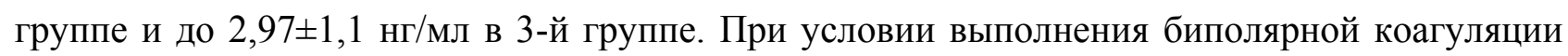

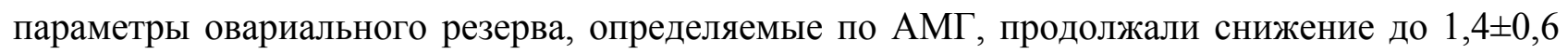


нг/мг (рис. 2).

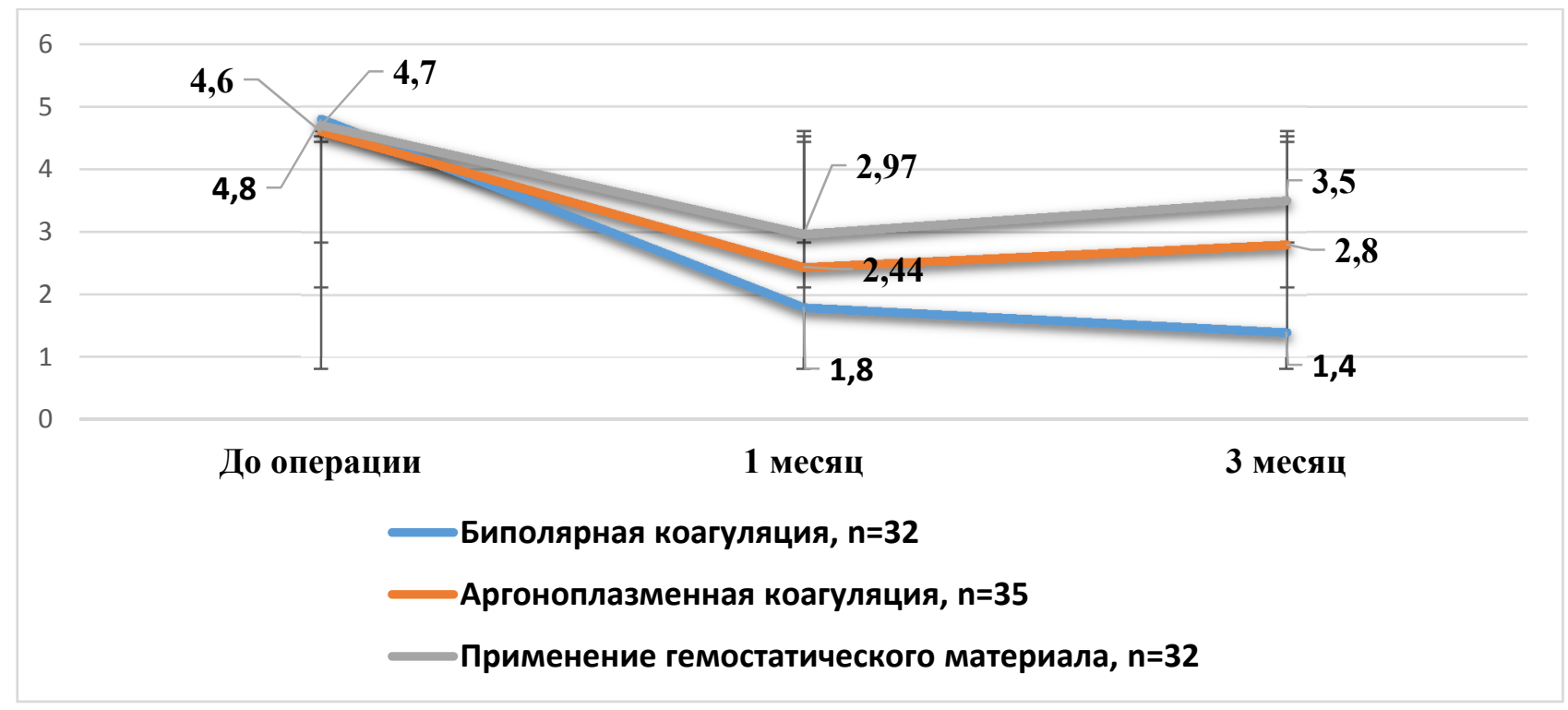

Рис. 2. Сравнительная характеристика динамики овариального резерва в послеоперационном периоде паџиенток с ЭКЯ в зависимости от метода эндохирургического гемостаза, $M \pm m$

С целью выяснения возможной взаимосвязи между снижением АМГ у пациенток позднего репродуктивного возраста после энуклеации кисты и параметрами ПОЛ/АОС перитонеальной жидкости в раннем послеоперационном периоде мы провели корреляционный анализ взаимоотношений процента снижения АМГ к 3-му месяцу послеоперационного периода и коэффициентом МДА/СОД. Процент снижения АМГ через 3 месяца после операции во всех клинических группах при прямой взаимосвязи сильно коррелировал с коэффициентом МДА/СОД ( $\mathrm{r}=0,89$ - 1-я группа, $\mathrm{r}=0,91$ - 2-я группа и $\mathrm{r}=0,88$ - 3-я группа). Коэффициент корреляции между ПВИ перитонеальной жидкости и процентом снижения АМГ также указывал на сильную прямую связь (r=0,86 - 1-я группа, $\mathrm{r}=0,81$ - 2-я группа и $\mathrm{r}=0,84$ - 3-я группа).

\section{Заключение}

Таким образом, в данном исследовании показано, что при энуклеации эндометриоидной кисты яичника усугубляется местный дисбаланс в системе цитокинов и ПОЛ/АОЗ, что может играть определяющую роль в глубоком снижении овариального резерва яичников у пациенток позднего репродуктивного возраста. При энергозависимом интраоперационном гемостазе на фоне регуляторной дисфункции, определяемой инертностью выработки IL2, на местном уровне нарушается сбалансированность функционального состояния межклеточных иммуномодуляторов IL6, TNF $\alpha$ их антагониста ИЛ-10, что ведет к усилению проапоптических влияний и замедлению регенеративно- 
восстановительных реакций. В результате усугубления дисбаланса в системе цитокинов и ПОЛ/АОЗ развиваются глубокие патофизиологические сдвиги, следствием которых становятся накопление токсических метаболитов, ишемический некробиоз в периоперационной зоне и нарушение тканевого ремоделирования. Данный процесс наиболее выражен при применении коагуляции биполярным электродом. Наименьшие сдвиги отмечены у пациенток позднего репродуктивного возраста после цистэктомии в системах ПОЛ/АОЗ и цитокиновом балансе и как следствие в снижении овариального резерва наблюдаются при щадящем методе применения интраоперационных гемостатиков в виде рассасывающегося гемостатического материала, изготовленного по методу контролируемого окисления восстановленной целлюлозы.

\section{Список литературы}

1. Гаспаров А.С., Дубинская Е.Д. Эндометриоз и бесплодие: инновационные решения. М.: МИА, 2013. 128 c.

2. Сахаутдинова И.В., Хабибуллина Р.Н. Оценка овариального резерва при эндометриоидных кистах яичников у женщин с нереализованной репродуктивной функцией // Здоровье и образование в XXI веке. 2017. №5. С.32-34.

3. Сахаутдинова И.В., Хабибуллина Р.Н., Кулешова Т.П. Тактика ведения пациенток репродуктивного возраста после цистэктомии по поводу эндометриомы на этапе амбулаторно-поликлинического звена // Журнал научных статей здоровье и образование в XXI веке. 2017. №6. С. 55-58.

4. Лоскутова Е.В., Воронцова И.А., Вахитов Х.М., Сафиуллин Т.Р. Роль дестабилизаторов процессов перекисного окисления липидов и антиоксидантной защиты в патогенезе гипоксии у недоношенных новорожденных // Казанский медицинский журнал. 2017. №5. T. 98. C.803-808.

5. Iwase A., Nakamura T., Nakahara T., Goto M., Kikkawa F. Assessment of ovarian reserve using anti-Müllerian hormone levels in benign gynecologic conditions and surgical interventions: A systematic narrative review. Reproductive Biology and Endocrinology. 2014. vol.12. no.1. P.125.

6. Vercellini P., Somigliana E., Vigano P., Abbiati A., Barbara G., Fedele L. Chronic pelvic pain in women: etiology, pathogenesis and diagnostic approach. Gynecol. Endocrinol. 2009. vol. 25. no. 3. P.149-158.

7. Соколова Е.И., Куценко И.И., Кравцов И.И. Состояние оксидантно-антиоксидантной системы перитонеальной жидкости и овариального резерва у пациенток с эндометриоидными кистами // Репродуктивный потенциал России: версии и контраверсии: 
тезисы XI Общероссийского научно-практического семинара. 2018. С. 61-62. 